\title{
La unidad de la conciencia en los comentadores griegos de Aristóteles
}

\author{
The unity of consciousness \\ in Aristotle's greek commentators \\ JESÚS DE GARAY SUÁREZ-LLANOS \\ Universidad de Sevilla
}

Recibido: 3/08/2017 Aceptado: 23/08/2017

\begin{abstract}
RESUMEN
Análisis de los comentarios griegos al De anima de Aristóteles, así como de otras interpretaciones neoplatónicas relativas a la conciencia sensible y la autoconciencia. En especial examen de las interpretaciones de Temistio, Filopón, Simplicio, Esteban, Plotino y Proclo. Breves indicaciones acerca de la recepción de los Elementos de teología de Proclo en Dietrich de Freiberg.

\section{PALABRAS CLAVE}

CONCIENCIA SENSIBLE; AUTOCONCIENCIA; NEOPLATONISMO TARDÍO; COMENTARIOS GRIEGOS AL DE ANIMA; DIETRICH DE FREIBERG
\end{abstract}

\begin{abstract}
Analysis of the Greek commentaries to Aristotle's De anima, as well as other Neoplatonic interpretations related to the sensitive consciousness and self-awareness. In particular, I focus on an examination of the interpretations of Themistius, John Philoponus, Simplicius, Esteban, Plotinus and Proclus. Brief indications about the reception of the Elements of theology of Proclus in Dietrich de Freiberg

\section{KEYWORDS}

SENSITIVE CONSCIOUSNESS; SELF-AWARENESS; LATE NEOPLATONISM; GREEK COMMENTS TO DE ANIMA; DIETRICH OF FREIBERG
\end{abstract}




\section{INTRODUCCIÓN}

DOS OBSERVACIONES PRELIMINARES:

$1^{\circ}$ ) "Grecia", la "filosofía griega clásica", "Platón y Aristóteles" si se quiere, no llegan a la filosofía moderna alemana directamente. La recepción de Grecia en Alemania es la recepción de un conjunto de transformaciones del aristotelismo y del platonismo.

En muchos aspectos, estas transformaciones de Platón y de Aristóteles se llevan a cabo en Roma entre el siglo I y VI. Ahora bien, la mayoría de los comentadores griegos de Aristóteles (en particular en Alejandría en el s.VI) son neoplatónicos. Y llevan a cabo una lectura neoplatónica de Aristóteles. Este aristotelismo neoplatónico es el que recibe la Modernidad, y Alemania en particular. Y no el Platón y Aristóteles que hoy se estudia en nuestras universidades.

$\left.2^{\circ}\right)$ Los inicios de la tradición filosófica alemana son medievales. Concretamente pueden reconocerse en el Studium Generale de la O.P. en Köln, regentada por Alberto Magno, que años más tarde dará lugar a la Universidad de Colonia.

Allí se forma una escuela filosófica, que con el paso de los años se autodenominará "albertista", claramente diferenciada del "tomismo". Casi la totalidad de los pensadores de esta escuela son alemanes: quizá los más conocidos son Meister Eckhart y Nicolás de Cusa.

Al final de estas páginas, mencionaré breve y esquemáticamente algunos rasgos de esta filosofía alemana medieval. Me referiré exclusivamente a uno de los autores de esta escuela medieval, Dietrich de Freiberg (Theodoricus Teutonicus), menos conocido pero que representa muy bien la orientación de esta tradición filosófica alemana. Esta filosofía alemana medieval recoge muchas de las doctrinas de ese aristotelismo neoplatónico de los comentadores griegos del siglo VI. En particular, señalaré algunos aspectos de la recepción de los Elementos de teología de Proclo en las doctrinas de Dietrich.

\section{Los COMENTARIOS AL DE ANIMA DE ARISTÓTELES}

Mi intención es mostrar de qué modo el neoplatonismo transforma la doctrina aristotélica acerca de la conciencia. Me ceñiré principalmente a los comentarios conservados ${ }^{1}$ al De anima de Aristóteles, con especial atención a lo relativo al capítulo $2^{\circ}$ del libro III.

1 Cfr. Themistius, On Aristotle On the Soul, R.B. Todd (ed.), London / Ithaca 1996 (Heinze V.3); Simplicius, On Aristotle On the Soul, J.O. Urmson (trad.) y P. Lautner (notas), London / Ithaca 1995 (Hayduck XI); Priscian, On Theophrastus on sense-perception, P.M. Huby (trad.) y J.O. Urmson (notas), London /Ithaca 1997 (Hayduck XI); "Simplicius", On Aristotle On the Soul 2,5-12, C. Steel y J.O. Urmson (trad.) y J.O. Urmson (notas), London / Ithaca 1997 (Hayduck XI); "Simplicius", On Aristotle On the Soul 3,1-5, H.J. Blumenthal (ed.), London / Ithaca 2000 (Hayduck XI); "Philoponus", On Aristotle On the Soul 3,1-8, W. Charlton (ed.), London / 
De los comentarios griegos disponibles al De anima, tres de ellos pertenecen a filósofos del siglo VI (Simplicio y Filopón, según la atribución tradicional recogida en los Commentaria in Aristotelem Graeca, editados por Hayduck), mientras que la de Temistio corresponde a mediados del siglo IV. En cuanto a la autoría, la de Temistio no está debatida, así como tampoco los comentarios de Juan Filopón a los dos primeros libros del De anima, ni el comentario denominado De intellectu, relativo a los capítulos 4 al 8 del libro III que sólo se conserva en la versión latina de Moerbeke (siglo XIII).

Sin embargo, hay otros dos comentarios al De anima escritos a lo largo del siglo VI, que sí plantean dudas en cuanto a la autoría. Uno de ellos es un comentario al libro III del De anima, atribuido tradicionalmente a Juan Filopón, que, sin embargo, es muy improbable que sea suyo. Actualmente el debate sigue abierto, pero la mayoría de los estudiosos (incluido el mismo Hayduck) asumen que el autor es Esteban de Alejandría. Recientemente, Peter Lautner ha argumentado que si bien es improbable que Filopón sea su autor, es posible que la autoría corresponda a otro discípulo de Filopón. A pesar de que el debate no está cerrado, sin embargo en lo sucesivo, en aras de la claridad de mi exposición, me referiré a Esteban como el autor de este comentario. Esteban es el último de los neoplatónicos de Alejandría, vive a finales del siglo VI, probablemente discípulo de Olimpiodoro en Alejandría, y es quien traslada la dirección de la Academia desde Alejandría a Constantinopla.

El otro comentario al De anima sobre el que el debate en torno a su autoría sigue abierto es el comentario tradicionalmente atribuido a Simplicio. Bossier y $\mathrm{Steel}^{2}$, en 1972, abogaron con numerosos argumentos a favor de Prisciano de Lidia como su autor. Este Prisciano fue uno de los que se exiliaron a Persia -junto con Damascio, Simplicio y otros- cuando Justiniano cerró la Academia de Atenas en el 529. Uno de las principales razones para excluir a Simplicio como su autor es una marcada diferencia de estilo en comparación con los otros comentarios del mismo Simplicio (por ejemplo a la Física o al De coelo). Mientas que en estos otros comentarios Simplicio ofrece una información prolija y precisa de sus argumentaciones, en este comentario al De anima no hay nada parecido. En cambio, si se compara este comentario al De anima con la Metaphrasis in Teophrastum de Prisciano, se observan abundantes similitudes tanto en estilo como en el contenido. La situación actual del debate es la siguiente: por una parte, hay un cierto acuerdo (con la importante excepción de

Ithaca 2000 (Hayduck XV); "Philoponus", On Aristotle On the Soul 3,9-13, W. Charlton (ed.), London /Ithaca 2000 (Hayduck XV); Philoponus, On Aristotle On the Intellect: De anima 3,4-8, F. Bossier (ed.), London / Ithaca 1991 (Hayduck XV).

2 F. Bossier y C. Steel, Priscianus lydus en de «in de Anima» Van pseudo(?)-Simplicius, Tijdschrift Voor Filosofie 34 (1972), 761 - 822. 
Ilsetraut $\mathrm{Hadot}^{3}$ ) de que Simplicio no es su autor. Por otra parte, la atribución a Prisciano no parece suficientemente firme, como ha mostrado recientemente Urmson. Sea como fuere, a efectos de la claridad de mi exposición, me referiré a Simplicio como el autor del comentario.

En resumen, disponemos de los siguientes comentarios al De anima de Aristóteles:

1.La paráfrasis de Temistio.

2.El comentario de Simplicio (atribuido por Carlos Steel a Prisciano). 3.El comentario de Filopón a los dos primeros libros del De anima.

4.El comentario de Filopón al libro III, capítulos 4 al 8, conocido como De intellectu.

5.El comentario de Esteban al libro III (atribuido tradicionalmente a Filopón).

Además de estos comentarios cuyos textos conservamos, hay otros dos comentarios perdidos, pero de los que disponemos de cierta información: el comentario de Alejandro de Afrodisias y el de Plutarco de Atenas. Ambos son muy citados entre los comentadores del siglo VI, y el de Alejandro también por Temistio. De Alejandro, además, conservamos -además de sus otros comentarios a otras obras de Aristóteles- sus escritos De anima y Mantissa, que supuestamente recogen buena parte de su interpretación del De anima de Aristóteles. En cambio, del comentario de Plutarco tenemos que conformarnos con las referencias de los comentadores del siglo VI, en particular de Filopón y Esteban.

Además de los comentarios perdidos de Alejandro y Plutarco de Atenas, existen ciertas dudas acerca de otros posibles comentadores del De anima, como Jámblico y Olimpiodoro. Sin embargo, hasta ahora, por los datos que disponemos, es improbable que escribieran esos comentarios.

Por último, y para cerrar estas notas introductorias, son muy numerosos los filósofos que ofrecen interpretaciones a las doctrinas del De anima de Aristóteles, ya desde Teofrasto. Obviamente la historia de la recepción del De anima en la Antigüedad es aquí inabarcable. A pesar de todo, hay algunos filósofos neoplatónicos que son constantemente citados por los comentadores del siglo VI como intérpretes de las doctrinas aristotélicas del De anima: concretamente Plotino, Porfirio, Jámblico, Proclo y Ammonio de Hermias. Por ello, a lo largo de mi exposición, me referiré ocasionalmente a ellos, pero sólo en la medida que dichos comentadores también lo hacen.

3 Simplicius or Priscianus? On the Author of the Commentary on Aristotle's "De Anima" (CAG XI): A Methodological Study, Mnemosyne 55, 2 (2002), 159-199 
Por ejemplo, el comentario de Juan Filopón a los dos primeros libros del De anima recoge las lecciones de Ammonio de Hermias, aunque con aportaciones personales del propio Filopón. Asimismo Simplicio afirma tener muy en cuenta la interpretación del De anima de Aristóteles llevada a cabo por Jámblico. Por otra parte, todos los comentadores del siglo VI recogen -habitualmente sin citarle- las interpretaciones que Proclo hace de Aristóteles en numerosos lugares. En cuanto a Plotino, es mencionado frecuentemente -muchas veces en tono crítico- como intérprete de Aristóteles.

\section{El NEOPLATONISMO DE LOS COMENTADORES}

En cuanto al contenido de estos comentarios, Temistio es una excepción, por su explícita adscripción aristotélica. Su interés se centra en explicar el texto del De anima, sin aludir a los pensadores neoplatónicos. El neoplatonismo era ya a mediados del siglo IV la corriente filosófica dominante, y sin duda Temistio conocía en alguna medida las doctrinas de Plotino y Porfirio, pero no parece tenerlas en cuenta. Es posible pero improbable que estuviera al tanto de las tesis de Jámblico. Sus referencias principales son Alejandro de Afrodisias y, en menor medida, Teofrasto. No obstante, algunas de las ideas neoplatónicas aparecen en su interpretación.

Aparte de Temistio, el resto de los comentadores (Simplicio, Filopón, Esteban) son claramente neoplatónicos. Como es sabido, entre los neoplatónicos existía la convicción de que Aristóteles y Platón coincidían en muchos puntos, aun cuando sus perspectivas fueran a veces diferentes. Por eso, en las academias neoplatónicas se estudiaba durante varios años concienzudamente toda la obra de Aristóteles antes de iniciarse en los textos platónicos. Consecuentemente muchas de las doctrinas aristotélicas están incorporadas de modo no problemático en las reflexiones de estos pensadores neoplatónicos. Y asimismo sus comentarios al De anima quieren reflejar la genuina doctrina de Aristóteles, aun cuando sus interpretaciones fuerzan en ocasiones los textos de Aristóteles para que armonicen con sus convicciones neoplatónicas.

Obviamente, no todos los neoplatónicos ofrecen las mismas interpretaciones de Aristóteles ni todos aceptan sin más esa supuesta armonía entre las doctrinas de Aristóteles y de Platón. Ya Plotino se mostró especialmente crítico respecto a Aristóteles, y otro tanto puede decirse de Siriano y de Proclo, quienes subrayan las discrepancias de Aristóteles con Platón. Pero, a pesar de todo, siempre permanece la certeza de que, por decirlo así, Aristóteles era esencialmente un pensador platónico más.

Esto implica en primer lugar un interés por aquellos textos de Aristóteles que más afectan a los planteamientos neoplatónicos. El mismo título de este escrito ("La unidad de la conciencia") refleja precisamente cómo una cuestión que en Aristóteles (y específicamente en el De anima) no ocupa el centro de 
los análisis aristotélicos, y sin embargo en los autores neoplatónicos pasa a un primer plano. Como es conocido, el neoplatonismo desarrolló notablemente la doctrina de Platón acerca de la importancia de la introspección ("conócete a ti mismo") y, más allá, de la reflexión sobre los datos de la conciencia como el método adecuado de la filosofía.

Por ello, desde Jámblico, al iniciarse en el plan de estudios que se seguía en las academias neoplatónicas, se comentaba el Alcibíades Primero atribuido a Platón, para subrayar la adecuación entre conocimiento de uno mismo y conocimiento del ser. La Sentencia 40 de Porfirio ${ }^{4}$ es en este sentido ejemplar: conocerse a sí mismo equivale al conocimiento del ser (40,30-31). Asimismo las palabras de Proclo al inicio de su comentario al Alcibíades son muy expresivas: "El principio de la filosofía y de la enseñanza de Platón es el conocimiento de sí (i் $\dot{\varepsilon} \alpha v \tau \tilde{\omega} \nu \gamma \nu \tilde{\omega} \sigma \imath \varsigma) ”(5,15-16)$.

Asimismo, en tanto el Principio primero de toda la realidad es concebido como Uno -y en esa misma medida cualquier realidad es en tanto que es una- la búsqueda de la unidad del alma humana presenta una particular importancia. La unidad de la conciencia humana representa así todo un programa filosófico para un pensador neoplatónico.

Por otra parte, desde Jámblico los filósofos neoplatónicos abordaban cual-

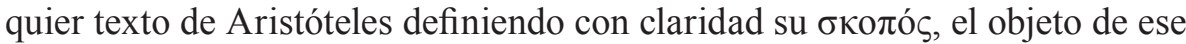
texto. En relación al De anima, hay una coincidencia en todos ellos de que su objeto es el alma racional humana. Por ello, afirman que en el De anima Aristóteles no analiza inteligencias ni almas superiores al ser humano, ni tampoco lo relacionado con la divinidad.

Esta caracterización del skopos del De anima por parte de los comentadores neoplatónicos tiene consecuencias, porque marca una clara discrepancia frente a Alejandro de Afrodisias, por una parte, y frente a Plotino por otra. Respecto a Alejandro, todos los comentadores neoplatónicos -y en este caso, también Temistio- rechazan la tesis de que el intelecto que hace todas las cosas (poietikos, en la terminología de Alejandro), al que Aristóteles alude en III-5, sea la misma divinidad. El argumento repetido por los comentadores neoplatónicos es que Aristóteles está analizando la racionalidad del alma humana, sin aludir a la divinidad ni a inteligencias superiores.

Por otra parte, frente a Plotino (y secundariamente también frente a Porfirio y Amelio), los comentadores neoplatónicos del siglo VI defienden la tesis de que "el alma humana ha descendido completamente", tal como Jámblico sostuvo frente a Plotino en su De anima, y tal como Proclo afirma en la proposición 211 de los Elementos de teología. A Plotino le atribuyen la tesis de que el alma humana no ha abandonado enteramente el mundo inteligible

4 Cfr. Porphyre, Sentences, L. Brisson (ed.), Vrin, París 2005. 
superior, por lo que el alma humana sigue manteniendo un vínculo esencial con esa Inteligencia superior, y en último término también con el Uno. Pero desde Jámblico todos coinciden en que la inteligencia del alma humana -en su actividad, e incluso en su esencia-, posee una especificidad independiente de las Inteligencias superiores. Por eso, por ejemplo, Esteban, al comentar III-5, considera la interpretación del nous poietikos de Plotino junto a la de Alejandro, y rechaza ambas por este mismo argumento: tanto Plotino como Alejandro introducen instancias ajenas a las facultades específicamente humanas para explicar la racionalidad humana.

En cualquier caso, el esfuerzo interpretativo mayor de estos comentadores radica en compaginar la doctrina aristotélica sobre la unión de alma y cuerpo con la doctrina platónica. Reconociendo la discrepancia entre ambos, a pesar de todo afirman que Aristóteles aceptó, no la separación, pero sí la separabilidad del alma respecto al cuerpo, así como su inmortalidad. Este punto es particularmente acentuado por Filopón quien lo reitera una y otra vez (reiteración explicable por la dificultad de compaginar esta doctrina con los textos aristotélicos).

Otras doctrinas neoplatónicas se introducen una y otra vez, como la estructura jerárquica entre las facultades -especialmente entre las distintas formas de racionalidad- o también la división tripartita o triádica característica de Proclo (entre lo imparticipado, lo participado y lo participante).

La introducción de Simplicio a su comentario es especialmente ilustrativa de estos intentos de compaginar los textos aristotélicos con posiciones neoplatónicas. Así Simplicio empieza su comentario con las siguientes palabras: "La primera y más importante cuestión es la verdad acerca de las cosas mismas, tanto acerca de otras realidades como acerca del alma, que es la más importante de todas para nosotros. Es por ello por lo que pienso que es necesario estudiar muy cuidadosamente el tratado Acerca del alma de Aristóteles". Y continúa señalando cómo Aristóteles desarrolló plenamente las intuiciones de Platón, tal como reconoció "ese excelente juez de la verdad que fue Jámblico" (1, 1-12). Y por ello, continúa Simplicio, explicará la obra de Aristóteles siguiendo la interpretación de Jámblico (1,1-20). En resumen, $1^{\circ}$ ) Hay que atenerse a la verdad de lo que es alma en sí misma. $2^{\circ}$ ) Aristóteles ha expuesto de la manera más completa lo que en Platón estaba solamente apuntado. $3^{\circ}$ ) Así lo ha reconocido Jámblico, que, según Simplicio, ha sido el mejor intérprete del De anima de Aristóteles, y cuya interpretación se propone seguir.

En cualquier caso, más allá de estas consideraciones preliminares, hay tesis aristotélicas que aparecen resaltadas con especial insistencia en estos comentarios. Una de ellas es la específica acepción de 'alteración' (alloiosis), cuando se refiere a los seres vivos. Aristóteles, efectivamente, en II-5, señala que el término 'padecer' (to paschein) tiene dos acepciones diferentes: “por 
un lado es una cierta destrucción por causa de la acción de un contrario; pero por otro lado es más bien la conservación (swteria) del ser en potencia por el ser en acto, de un modo semejante a como la potencia es con relación al acto: pues ejerciendo la ciencia se tiene la ciencia. Y este cambio o no es una alteración (pues es un progreso hacia sí y hacia el acto: epidosis eis auto kai eis entelecheian) o bien constituye otro género de alteración" (417b 2-7). Es decir, concluye Aristóteles: "hay dos clases de alteración: una es el cambio (metabolen) hacia las disposiciones privativas, la otra es el cambio hacia los hábitos (hexeis) y la naturaleza" (15-16). En síntesis, Aristóteles afirma que las actividades de los seres vivos difiere de los otros tipos de movimiento, en tanto que la actividad del viviente procede de sí mismo y tiene un sentido de perfeccionamiento o desarrollo de lo que ya estaba en potencia.

Pues bien, los comentadores a los que vengo refiriéndome, incluido Temistio, insisten de una forma casi obsesiva en este punto, precisamente para destacar la autonomía del viviente. De este modo, la actividad sensorial e intelectual no tiene un carácter pasivo, no es propiamente un padecer, sino que es una actividad que procede del propio viviente, es decir, del alma. En el caso de los comentadores neoplatónicos, esta insistencia se traduce en la afirmación de la independencia del alma con respecto al cuerpo. Los órganos sensoriales sí padecen la acción de los objetos externos (y aún aquí establecen numerosas reservas, especialmente Filopón), pero la actividad sensorial procede exclusivamente del alma. Y en un grado mayor, esto vale también para la imaginación y sobre todo para la inteligencia. Ciertamente la inteligencia es impasible, como dice Aristóteles. Pero ellos subrayan que también, aunque en un grado menor, es impasible toda la actividad sensorial.

\section{LA CONCIENCIA SENSIBLE}

Lo relativo a la unidad de la conciencia es analizado por los comentadores primariamente al comentar el capítulo $2^{\circ}$ del libro III. No obstante, el texto aristotélico no es interpretado del mismo modo por todos ellos. La interpretación a la que todos remiten en primer lugar es la de Alejandro de Afrodisias, quien en su tratado Acerca del alma atribuye la conciencia sensible al sentido común (koine aisthesis): "Con este sentido común nosotros sentimos también que estamos viendo y oyendo y percibiendo con cada uno de los sentidos particulares; porque quien ve tiene la sensación de que está viendo, y quien oye, de que está oyendo. Es, en efecto, cierto que no es con otra facultad distinta del sentido con la que nosotros tenemos la sensación de que estamos percibiendo: ciertamente, no vemos que vemos, ni oímos que oímos, pues ni el ver es visible, ni el oír audible, sino que esta actividad pertenece al sentido primero y soberano,

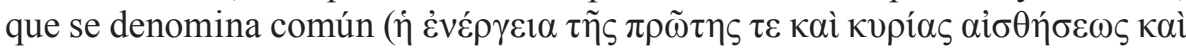




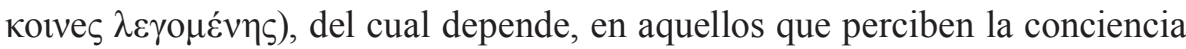

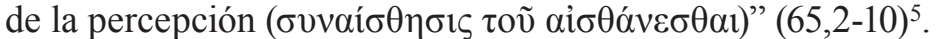

Propiamente Aristóteles no utiliza la expresión 'sentido común' (koine aisthesis) en el De anima. Aunque sí en el tratado Acerca del sueño y la vigilia. Incluso parece que, más bien, en el De anima reconoce que cada sentido externo no sólo conoce su sensible propio, sino además es consciente de su propia sensación. Dejando ahora de lado cuál pueda ser la correcta interpretación del texto aristotélico, la interpretación de los demás comentadores no siempre coincide con la de Alejandro.

Así, Temistio sigue detalladamente las argumentaciones de Aristóteles: en primer lugar, habría que proceder al infinito si hubiera otro sentido que fuera consciente de la sensación; y a su vez otro, y así sucesivamente; en segundo lugar, el acto de ver percibe no sólo el color sino también la oscuridad, por lo que percibe tanto el ver como el no ver; pero en tercer lugar y sobre todo -y éste es el principal argumento que recogerán los comentadores al referirse a la conciencia-, la vista ve el color pero también percibe el acto de ver, porque, tal como afirma Aristóteles, "el acto de lo sensible y el del sentido son uno y el mismo, si bien su esencia no es la misma" (425b25-27). Es decir, aunque el objeto visto y la visión sean esencialmente realidades diferentes, sin embargo el acto de ver es uno solo, e integra simultáneamente tanto el acto de ver como el objeto visto. Por ello, no es posible percibir el objeto sin percibir simultáneamente el acto de ver. Obviamente el argumento es objetable, pero Temistio y los demás comentadores lo aceptan como el argumento principal por el que los sentidos no sólo conocen su objeto propio sino también son conscientes de su actividad.

Este argumento se repite una y otra vez también al tratarse de otras actividades psíquicas, y especialmente al referirse a la inteligencia. En general es uno y el mismo el acto de conocer y el objeto conocido, aun cuando en su esencia sean distintos. En potencia son realidades diferentes, pero en el acto de conocer son un solo acto que integra la diferencia entre actividad de conocer y objeto conocido. Por eso, el argumento es utilizado no sólo para la conciencia sensible sino en general para cualquier actividad psíquica. Por eso, ilustra Filopón en De intellectu, entender algo implica siempre entenderse (21,3-4).

Temistio continúa glosando el texto aristotélico explicando que los cinco sentidos están unidos en un único sentido que es como el centro donde convergen y de donde proceden todas las líneas de la circunferencia. Es a la vez divisible e indivisible, y en esa medida discrimina simultáneamente desde su unidad todas las diferencias de los diferentes sentidos y las propias actividades sensitivas (159,8-22). Los demás comentadores abundan en esta interpretación,

5 Acerca del alma; Acerca del destino, J.M. García Valverde (ed.), Gredos, Madrid 2013. 
considerando que los cinco sentidos tienen su raíz en una primaria facultad sensorial, denominada generalmente "sentido común" por los comentadores (con la excepción de Temistio, que evita la denominación de 'sentido común'). Temistio, por lo demás, caracteriza el pneuma como el órgano específico de esta primaria facultad sensorial $(159,23-160,19)$, de la que derivan como de una fuente todos los demás órganos sensoriales.

En resumen, para los comentadores griegos, la unidad de la conciencia sensible viene dada por una única facultad sensorial (la koine aisthesis) que integra todos los sentidos externos, y es capaz de discriminar los sensibles propios de cada sentido y también las actividades correspondientes. Esta facultad pasa de la potencia al acto gracias a la propia actividad del alma, sirviéndose como un instrumento de las impresiones sensibles recibidas en los órganos sensoriales. Esta conciencia sensible es previa a la actividad de la imaginación, en tanto que se da únicamente en el momento en que la sensación es actual, es decir, sólo se da cuando el objeto sensible está presente, a diferencia de lo que sucede en la imaginación.

No obstante, los comentadores neoplatónicos -al explicar esta unidad de la conciencia sensible, que reside primariamente en el sentido común- añaden una observación que obviamente no es aristotélica. En concreto, tanto Esteban como sobre todo Simplicio, señalan que si el sentido común es capaz de conciencia, es porque participa de la razón. Y otro tanto valdría para cada uno de los cinco sentidos: si son capaces de conciencia, es porque son en cierta manera racionales.

«Percibir que percibimos, indica Simplicio, me parece que es algo sólo propio del hombre. Porque la conversión sobre sí mismo ( una actividad de la vida racional. Esto se muestra porque nuestra racionalidad

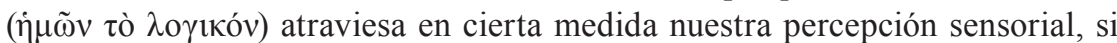
es que la facultad sensorial humana es capaz de conocerse a sí misma ( $\dot{\varepsilon} \alpha v \tau \tilde{\eta} \varsigma$ $\dot{\alpha} v \tau \imath \lambda \eta \pi \tau \imath \kappa \eta ́)$. Pues en cierto modo lo que percibe se conoce a sí mismo, percibiéndose a sí mismo mientras conoce. Y por ello, reflexiona sobre sí mismo

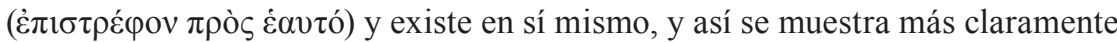
separado de los cuerpos, en tanto que todo cuerpo, teniendo diferentes partes de sí mismo en diferentes lugares, nunca sería capaz de volver su atención hacia sí

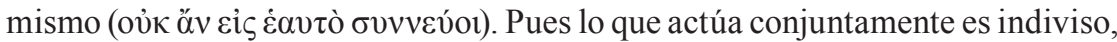
mientras que todo cuerpo está dividido. Por tanto, nuestra facultad sensorial es

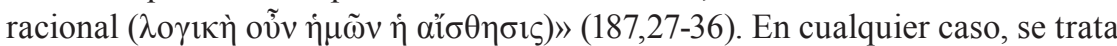
de percepción sensorial, en tanto que se refiere a lo individual, y la razón no conoce lo individual $(188,11-12)$.

Simplicio explica $(192,12-20)$ cómo la facultad sensorial proyecta su forma sobre la impresión recibida en el órgano sensorial. Es el alma sensitiva la que 
actúa proyectando desde sí misma ( $\dot{\varepsilon} \alpha v \tau \tilde{\eta} \varsigma$ ) su propia forma racional sobre las impresiones recibidas. La proyección (probole, proballein) es la actividad característica del alma que actúa desde su propia actividad. "El alma sensitiva se despierta proyectando sus propias

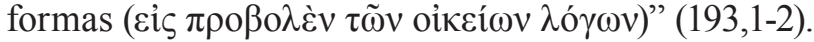

En tanto que la koine aisthesis humana es capaz de conciencia, en esa medida resulta equívoco referirse a ella como similar a la koine aisthesis de los animales irracionales. Simplicio remite a Jámblico para reforzar esta afirmación: "Tal como Jámblico dice, nuestra facultad sensorial es homónima con

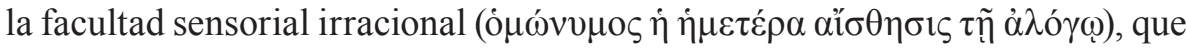
es aquella que se inclina como un todo hacia el cuerpo, pero no vuelve sobre sí misma, como [por el contrario sí lo hacen] el intelecto o la razón” (187,37-188,1).

En cualquier caso, continúa Simplicio, la facultad sensorial no tiene suficiente capacidad para reflexionar enteramente sobre sí misma: no puede conocer su esencia ni su poder sino sólo tiene conciencia de su actividad, y sólo en el

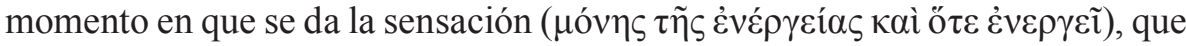
es cuando está presente el objeto sensible (188,1-3).

Lo que vale para la facultad sensorial en su conjunto (es decir, para el sentido común), vale igualmente para cada uno de los cinco sentidos -afirma Simplicio- ya que el sentido común no tiene ningún objeto propio sino que actúa solamente a través de cada uno de los sentidos. Es decir, cada uno de los sentidos lleva a cabo una actividad que es también racional y consciente. La conciencia sensible no es un atributo específico del sentido común sino que hay conciencia sensible en cualquier sensación.

Esteban, por el contrario, a diferencia de Simplicio, no es tan complaciente con Aristóteles: Aristóteles, dice, no se expresa correctamente cuando atribuye conciencia a la facultad sensorial $(466,46-47)$. Es absurdo, señala, que el mismo sentido conozca que ve, porque implica capacidad de volver sobre sí mismo (epistrephousa). Pero, continúa Esteban, si el sentido vuelve sobre sí mismo, entonces es una actividad separada de la materia, y por consiguiente pertenece a una sustancia que es eterna e incorpórea, lo cual, insiste, es absurdo, porque los sentidos no son eternos (466,39-48). No obstante, Esteban concede que si la actividad sensible implica conciencia, ello se debe exclusivamente a que es

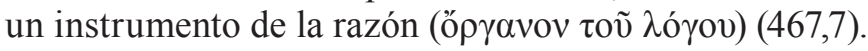

La conclusión en ambos casos (Simplicio y Esteban) viene a ser la misma: la conciencia es un atributo de la racionalidad. Si hay conciencia sensible, dirá Simplicio, es sólo en tanto los sentidos participan de la razón. Esteban afirmará lo mismo, pero justamente negando que los sentidos como tales sean conscientes de su propia actividad. 


\section{CONCIENCIA RACIONAL Y AUTOCONCIENCIA ${ }^{6}$ \\ V.1. ARISTÓteLES: DE ANIMA, III-4}

Así pues, mientras que Temistio -como Alejandro o Teofrasto antes- acepta que la conciencia es un atributo específico de la facultad sensorial, y afirma que ésa es la doctrina genuina de Aristóteles, por otro lado Simplicio y Esteban aceptan la existencia de la conciencia sensible pero sólo en tanto es la razón la que posibilita esta conciencia sensible, interpretando así de un modo nuevo los textos de Aristóteles.

En cualquier caso, la cuestión de la unidad de la conciencia no termina en el análisis de la conciencia sensible, sino que se plantea nuevamente al examinar la conciencia intelectual, tal como afirma el propio Aristóteles (III-4, 429b 6-10: "cuando el intelecto ha llegado a ser cada uno de sus objetos a la manera en que se ha dicho que lo es el sabio en acto [...], entonces el intelecto es capaz también de entenderse a sí mismo"'. O también más adelante (430a23): "el intelecto es inteligible exactamente como lo son sus objetos inteligibles".

Sobre este punto, todos coinciden en una idea central. En palabras de Filopón (De intell., 21,3-18 ), que suscriben todos los demás, entender algo implica siempre entenderse. No es posible entender sin entenderse a sí mismo.

\section{V.2. TEMISTIO: EL YO COMO INTELIGENCIA}

En cualquier caso, la cuestión concreta que una y otra vez se plantean estos comentadores es dónde radica el "yo" (to egó). Esta es una cuestión que, como hemos visto, Plotino formula desde una perspectiva claramente neoplatónica, pero que también encontramos en Temistio, desde una perspectiva atenta a la literalidad del texto aristotélico.

Afirma Temistio: "Dado que en todo lo que está combinado de lo potencial y lo actual, son distintos la cosa particular (tode ti) y la esencia de tal cosa particular, así también serán distintos el 'yo' y la esencia del 'yo' (

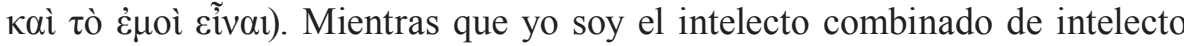

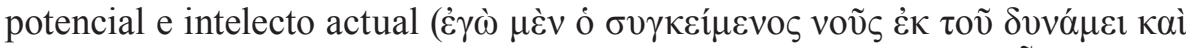

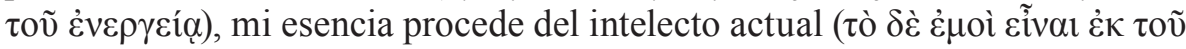

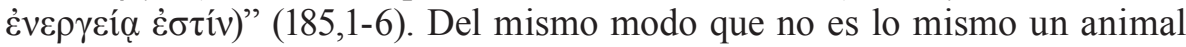
individual y la esencia de ese animal, asimismo tampoco es lo mismo el yo y la esencia del yo (185,15-18).

Es decir, según Temistio, lo que determina al 'yo' es el intelecto activo: "nosotros somos el intelecto activo (hemeis ho poietikos nous)" (186,3), afirma

6 Cfr. R. Sorabji, The Philosophy of the Commentators 200-600 AD, vol. 1, Ithaca / New York 2005, pp. 145-158.

7 Acerca del alma, T. Calvo (ed.), Gredos, Madrid 1994. 
reiteradamente (186, 14-16; 190, 18-19). Más aún, Temistio aclara que éste es precisamente el sentido del texto aristotélico. Este intelecto activo tiene una realidad que trasciende los individuos, pero que se manifiesta en cada uno de nosotros y es el que unifica toda la actividad del yo. Temistio lo compara al sol, que existe en sí mismo, pero que se multiplica con sus rayos. Del mismo modo, el intelecto activo se multiplica en cada uno de nosotros como iluminaciones diferentes (191,11-16). Este intelecto activo ha de ser el mismo para todos los hombres, porque de otro modo no sería posible la comunicación entre los seres humanos, ni que un maestro enseñase al discípulo. Además quedaría sin explicar de dónde proceden las nociones primeras (hai koinai ennoiai), las primeras definiciones y los primeros axiomas (191,17-24).

No obstante, el yo concreto que cada uno somos implica también al intelecto potencial y no sólo al intelecto activo. Ambos intelectos, potencial y activo, son separados, sin mezcla e impasibles, siendo el activo anterior por naturaleza, aunque no según el tiempo (195,18-21).

En cualquier caso, caracterizar al 'yo' como intelecto activo y como combinación de intelecto activo y potencial no es suficiente para Temistio, en tanto que son separados y trascienden la singularidad empírica de cada uno de nosotros. Por eso, Temistio afirma que aquello por lo que razonamos discursivamente, amamos y odiamos es el intelecto denominado "común" (koinos), o también "pasivo (pathetikos): 186,8-9". Este intelecto común está, dice, claramente indicado por Aristóteles en I-4, 408b 18-30: "Discurrir, amar, odiar no son, por lo demás, afecciones de la intelección, sino del sujeto que lo posee en tanto que lo posee. Esta es la razón de que, al corromperse éste, ni recuerde ni ame: pues no eran afecciones de aquél, sino del conjunto ( $\dot{\alpha} \lambda \lambda \dot{\alpha}$

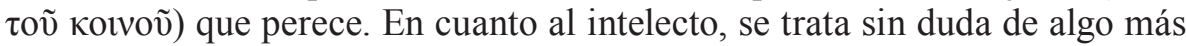
divino e impasible".

Así pues, el yo, en tanto que sujeto que razona, que ama y odia, es el nous pathetikos, que se corrompe al morir el sujeto. Así concluye Temistio su comentario a III-5: " De todo esto resulta evidente que nosotros estamos en lo cierto cuando sostenemos que, por una parte, hay un intelecto pasivo y perecedero (pathetikon noun kai fzarton), al cual también denomina 'común' y que es inseparable del cuerpo, y debido a la mezcla, Teofrasto dice que se produce el olvido y la confusión. Y que, por otra parte, hay otro intelecto que es como una combinación de lo actual y lo potencial, del cual ellos (Aristóteles y Teofrasto) afirman que está separado del cuerpo, que es imperecedero e ingénito: estos intelectos (actual y potencial) son en cierto modo uno y en cierto modo también dos, pues uno es lo que está compuesto de materia y forma" $(200,10-19)$.

Aunque no sea posible ahora analizar más detenidamente la confusa propuesta de Temistio, sin embargo su explicación curiosamente no resulta tan alejada de la de Plotino como pudiera parecer a primera vista, dada la marcada 
diferencia terminológica y conceptual. En ambos casos, su concepción del 'yo' procede de una instancia superior (la hipóstasis de la Inteligencia en el caso de Plotino; y en Temistio el Intelecto activo separado que difunde su luz sobre los intelectos activos individuales). Pero además ese 'yo' sólo se muestra cuando se integra en una facultad "inferior": la imaginación en el caso de Plotino, el nous pathetikos en Temistio. Lo cual es significativo porque, de hecho, entre los comentadores neoplatónicos de Aristóteles -y muy explícitamente en Filopónfrecuentemente se alude a la equivalencia entre nous pathetikos e imaginación.

\section{V.3. Plotino: CONCIENCIA E IMAGINACIÓN}

Sin embargo, entre los comentadores neoplatónicos, el asunto recibe un desarrollo nuevo, sobre todo a partir de Plotino, quien plantea la cuestión de la conciencia no solamente a nivel de la actividad sensible sino también la conciencia de nuestros deseos y en general de toda nuestra actividad psíquica.

Para Plotino, la conciencia intelectual presenta un especial interés porque -como ya mencioné más arriba- afirma que el alma humana sigue unida al mundo inteligible, y por tanto a la Inteligencia (con mayúscula), que es la hipóstasis que está por encima del Alma. Esta doctrina -reconoce Plotino- es una innovación que otros muchos platónicos no compartirán (4.8 [6] 8, 1-11). Y efectivamente desde Jámblico los neoplatónicos de los siglos V y VI rechazarán esta tesis.

Pero a Plotino se le plantea el problema de cómo es posible que: 1) si la inteligencia entiende siempre, tal como Aristóteles afirma en III-5 ("no ocurre, desde luego, que el intelecto entienda a veces y a veces deje de entender": 430a 22); 2) y si el alma humana posee inteligencia; 3) cómo es posible entonces que nosotros no tengamos conciencia de esa intelección ininterrumpida. Si la inteligencia está en el alma, entonces deberíamos entender siempre de modo continuado. Pero de hecho no es así.

La solución adoptada por Plotino es la siguiente: "No todo cuanto hay en el alma es ya por eso perceptible conscientemente ( $\alpha i \sigma \theta \eta \tau$ tóv), sino que nos llega a nosotros sólo cuando nos entra en la percepción sensible. Ahora bien, cuando una cualquiera de las partes que están activas no se comunica a la facultad sensitiva, todavía no ha atravesado el alma entera. Así pues, no tenemos

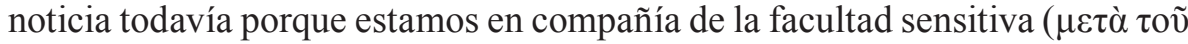

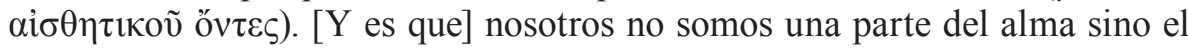
alma entera. Y además, cada una de nuestras potencias psíquicas, viviendo siempre, ejercita siempre por sí misma su propia actividad. Pero el tener noticia de ello se produce cuando hay comunicación y aprehensión (antílepsis). Por tanto, si ha de haber percepción de las potencias así presentes en nuestra alma, 
es preciso también volver (epistrephein) hacia dentro la facultad perceptiva y obligarla a aplicar su atención (prosoché) a aquello interior"s (5.1 [10] 12, 5-14).

En síntesis, Plotino acepta que la inteligencia del alma humana entiende siempre, pero reconoce también que no somos siempre conscientes de esa actividad intelectual ininterrumpida. La causa, explica Plotino, es que no somos sólo inteligencia sino también sensibilidad: la unidad del alma integra a la sensibilidad con la inteligencia. Pero la unidad de la conciencia exige que intervenga la totalidad de las partes del alma. Es decir, mientras que la actividad intelectual -que es siempre autoconciencia- no se expresa de un modo sensible, mientras tanto no hay conciencia. Sólo cuando la actividad intelectual es percibida sensiblemente, sólo entonces hay conciencia de sí. Y concretamente, añade Plotino, el lugar propio de la sensibilidad donde aparece la conciencia intelectual es en la imaginación, en tanto que es el lugar de la sensibilidad donde la inteligencia proyecta su actividad. De este modo, la imaginación -la imaginación superior, en tanto que Plotino distingue dos tipos de imaginación, superior e inferior- es el lugar propio de la conciencia de sí. De modo anacrónico, se podría decir que para Plotino el "yo empírico" tiene su lugar propio en la imaginación.

De hecho, señala Plotino (1.4 [46] 10, 6-29), tenemos experiencia de actividades teóricas y prácticas en las que nos olvidamos completamente de nosotros mismos, como cuando leemos con especial concentración o en determinadas acciones valerosas. Entonces la inteligencia se ejerce sin imaginación, y por ello no tenemos conciencia (parakolouthein) de nosotros mismos.

\section{V.4. ESTEBAN DE ALEJANDRÍA: INTELIGENCIA HUMANA Y ATENCIÓN}

Esteban recoge esta doctrina de Plotino, caracterizándola como una de las principales interpretaciones de los textos aristotélicos sobre la conciencia. Esteban explica que efectivamente no entendemos siempre porque el alma humana se ocupa de otras muchas realidades y sólo ocasionalmente reflexiona sobre sí misma. Desde luego, cuando se ocupa de realidades corporales se olvida de sí misma. Pero en general, dice Esteban, sucede como cuando nos encontramos con alguien importante acompañado por un amigo nuestro: saludamos a esa persona famosa en primer lugar sin apenas atender a ese amigo, porque estamos muy familiarizados con él. Algo así sucede con la presencia de la inteligencia a sí misma con la que estamos tan familiarizados que normalmente no le prestamos atención.

No obstante, aunque Esteban aprueba que Plotino limite su análisis al alma humana, sin embargo rechaza que la inteligencia humana esté siempre en actividad aunque sea inconsciente. Por el contrario, lo específico de la inteligencia

8 Enéadas, J. Igal (ed.), Gredos, Madrid 1998. 
humana -no como imaginación sino como inteligencia- es que su actividad es intermitente $(538,33-539,10)$.

Las palabras de Aristóteles de que el intelecto entiende siempre efectivamente se refieren al intelecto humano, pero en el sentido -dice Esteban- de que siempre hay hombres que están entendiendo: no quiere decir que Sócrates siempre entiende sino que siempre hay algún hombre que está entendiendo en el universo en su conjunto.

En cualquier caso, las interpretaciones de Temistio y Plotino no son las únicas. Entre los comentadores de Aristóteles se mencionan otras explicaciones de la conciencia intelectual, situándola por ejemplo en la doxa (solución que algunos atribuyen a Plutarco de Atenas) o en el logos (por ejemplo Proclo en su comentario al Timeo).

En el siglo VI hay una propuesta que adquiere importancia, que se refiere a la atención (he prosoché, to prosektikón), concebida como una facultad específica de la conciencia intelectual. Es decir, la instancia desde la cual se expresa el 'yo' no sería ni el sentido común, ni la imaginación, ni el nous pathetikos, ni el intelecto activo, sino la atención. Es decir, según esta tesis, la conciencia pertenece a la atención, que sería una facultad diferenciada de las mencionadas por Aristóteles. La atención había sido objeto de análisis por diversos autores previamente como Filón de Alejandría, y después por Epicteto, que dedica una de sus Disertaciones precisamente a la prosoche. Sin embargo, ahora en estos autores neoplatónicos significa directamente conciencia intelectual.

A la atención se refiere explícitamente Esteban, cuando analiza las diversas explicaciones que se han dado acerca de la conciencia sensible. Así, después de revisar las opiniones de Alejandro y de Plutarco de Atenas, alude a algunos intérpretes recientes, cuya propuesta él mismo defiende. Quiénes sean estos intérpretes Esteban no lo dice, pero se puede reconocer entre ellos a Proclo, Damascio y probablemente Olimpiodoro, ya que ellos aluden explícitamente a la atención como una facultad diferenciada que determina la conciencia del yo.

Así, en Damascio, en su Comentario al Fedón, encontramos referencias a la atención (to prosektikon), en tanto posibilita orientarse a uno mismo en diferentes direcciones, gracias a que la atención es conciencia (epistasis) acompañada de reflexión (meta epistrophes), a diferencia de otras formas de conocimiento, que se dirigen directamente a los objetos (269-272). Se trata de una facultad junto a otras, que actúa como un testigo respecto a las otras, como conciencia (suneidos) respecto a las facultades apetitivas, y como prosektikon respecto a las cognitivas. Esta distinción entre suneidos y prosektikon está también repetida por Damascio en este mismo Comentario al Fedón, 465, 11-16, y más tarde también por Olimpiodoro, en su Comentario al Alcibíades (23,16-17).

La función de la atención la resume Esteban en su comentario a III-2, precisamente para rechazar la tesis de Aristóteles y de Alejandro de que el 
sentido común sea el responsable de la conciencia sensible: "Recientes intér-

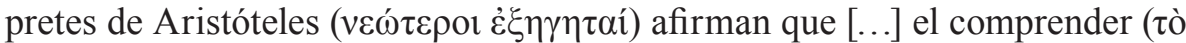
$\grave{\alpha} v \tau \imath \lambda \alpha \mu \beta \alpha ́ v \varepsilon \sigma \theta \alpha \imath)$ las actividades de los sentidos pertenece a la parte del alma

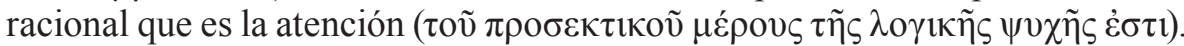
Pues, según ellos, el alma racional no posee sólo cinco facultades: voũ $\varsigma, \delta i \alpha$ -

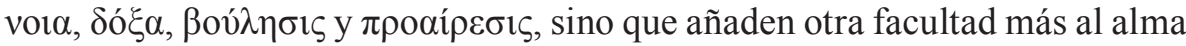
racional a la que denominan atencional (

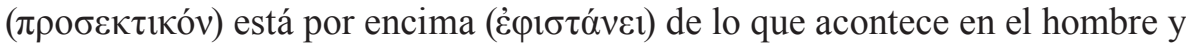
dice 'yo pienso', 'yo razono', 'yo opino', yo me irrito', 'yo deseo'. En general,

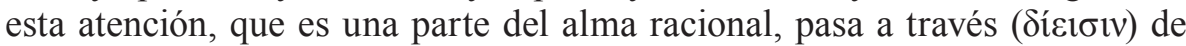
todas las facultades, las racionales, las irracionales y las vegetativas. Así pues, dicen, si necesariamente la atención ( las facultades, también atravesará los sentidos, de modo que avance y diga: 'yo veo', 'yo oigo'. Pues es propio del que aprehende las actividades el decir tales cosas. Si por consiguiente es la atención la que dice estas cosas, entonces es ella la que capta las actividades de los sentidos. Pues es preciso que sea uno (๕̊v) el que comprenda las actividades de todas las facultades, puesto que también es uno el hombre. Pues si uno captase unas, y otro otras, sería semejante -tal como dice Aristóteles en otros lugares (III-2, 426b19-20) - a como si tú percibieras esto y yo aquello. Por tanto, es preciso que sea uno, y eso uno es la atención ( $\tau$ ò $\pi \rho \circ \sigma \varepsilon \kappa \tau 1 \kappa o ́ v)$. Pues esta atención va y viene sin cesar ( $\varphi$ oı $\tau \tilde{\alpha})$ a través de todas las facultades cognoscitivas y vitales ( $\tau \tilde{\omega} v \gamma v \omega \sigma \tau 1 \kappa \tilde{\omega} v \kappa \alpha i ̀ \tilde{\omega} v \zeta \omega \tau 1 \kappa \tilde{\omega} v)$. Ahora bien, si va y viene a través de las facultades cognoscitivas, se denomina $\tau$ ò

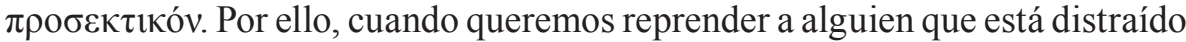
con relación a actividades cognoscitivas, le decimos: ‘Presta atención!' ( $\pi \rho o ́-$ $\sigma \varepsilon \chi \varepsilon \sigma \alpha v \tau \tilde{\omega})$. Por el contrario, si avanza a través de las actividades vitales, se denomina $\sigma v v \varepsilon 1 \delta o ́ \zeta ;$ por ello, la tragedia alude a la conciencia ( $\dot{\eta} \sigma u ́ v \varepsilon \sigma 1 \zeta)$, 'porque tengo conciencia ( $\sigma u ́ v o t \delta \alpha$ ) de haber realizado acciones terribles'. Por

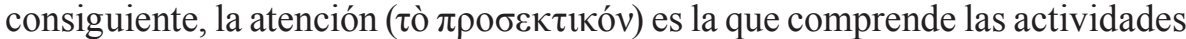
de los sentidos". Y concluye así Esteban: "Por tanto, no habla correctamente Aristóteles [cuando se refiere a la facultad sensorial como sede de la conciencia sensible], sino que, tal como decimos, pertenece a la atención (prosektikon) del alma conocer las actividades de los sentidos".

$$
\begin{aligned}
& \text { VI. La autoconciencia en Proclo (s.V D.JC) } \\
& \text { Y SU Recepción en Dietrich de Freiberg (s.XIII) }
\end{aligned}
$$

\section{VI.1. PROCLO:}

Proclo tiene una especial relevancia entre los intérpretes griegos de Aristóteles, porque su influencia es grande entre los comentadores aristotélicos del siglo VI, y porque la recepción latina de sus Elementos de teología (1268) 
- asociada a la relectura del Liber de causis- define una nueva interpretación de Aristóteles entre los discípulos alemanes de Alberto Magno.

Tal como se acaba de mencionar, en Proclo aparece, quizá por primera vez,

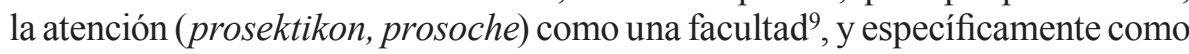
la facultad de la conciencia (parakolouthoûn) ${ }^{10}$, es decir como un acompañar consciente al conocimiento de objetos y en general a otras actividades del alma.

No obstante, la autoconciencia como tal (el yo, podríamos decir) es un rasgo específico de la inteligencia (nous). Así se afirma rotundamente en las proposiciones 167 y $168^{11}$ : todo intelecto se entiende a sí mismo; todo intelecto en el acto de intelección sabe que entiende; no hay disparidad entre el intelecto que entiende y el intelecto que sabe que entiende ${ }^{12}$. Según Proclo, la circularidad es un rasgo que define a todo lo real, porque todo aquello que procede de algo y hacia ello se vuelve tiene una actividad circular (Elth, 33). Pero lo característico de la inteligencia es que es capaz de conocer no sólo su actividad aquí y ahora, sino que es capaz de conocer su esencia: Todo aquello que es capaz de conocerse a sí mismo es capaz de conversión completa hacia sí mismo (panthei epistreptikon estin): Elth, $83^{13}$. En esta misma medida, la inteligencia se constituye a sí misma: es autoconstituyente (authypostaton): Elth, 40-51.

Ahora bien, fiel a la doctrina neoplatónica, Proclo subraya que más allá de la inteligencia está la unidad. Es decir, que la autoconciencia no es la que da unidad al ser humano, sino algo previo a la inteligencia autoconsciente: "Quizá no es lo mismo 'la flor del intelecto' y la 'flor de toda alma humana', sino que la flor del intelecto es lo más unitario de nuestra vida intelectiva, mientras que la flor del alma humana es lo 'uno' de todas las facultades del alma, facultades que son de muchas formas. Pues nosotros no somos sólo intelecto, sino asimismo razonamiento, opinión, atención (prosoché), elección y, con anterioridad a estas potencias, somos una esencia una y múltiple, divisible e indivisible"14. Es decir, hay algo uno en nosotros (la "flor del alma humana") anterior a la unidad de la inteligencia (la "flor del intelecto").

En un sentido cercano puede interpretarse otro texto de su Comentario al Parménides (957,35-958,11): "Existe además cierta vida una que mueve al alma hacia todas estas cosas, por virtud de la cual decimos 'yo deseo (egó epithumô)',

9 Cfr. Oráculos Caldeos, F. García Bazán (ed.), Gredos, Madrid 1991, p. 121

10 Cfr. la referencia de Damascio al comentario de Proclo a las Enéadas en In Phaed,p.271.

11 Cfr. Proclus. The Elements of Theology, E.R. Dodds (ed.), Oxfrod 193; Elementos de teología, J.M. García Valverde (ed.), Trotta, Madrid 2017.

12 Cfr. Liber de causis, XIII, 109: omnis intelligentia intelligit essentiam suam

13 Cfr. Liber de causis, XV: omnis sciens qui scit essentiam suam est rediens ad essentiam suam reditione completa.

14 Cfr. Oráculos Caldeos, p. 121 
'yo me irrito (egó thymoûmai)', o 'yo eligo tal o tal (egó proairoûmai)'. Porque este principio vital consiente ( $\sigma 0 v \varepsilon \pi v v \varepsilon v ́ \varepsilon \imath$ ) con estas actividades y vive con ellas, siendo un poder que dirige su impulso (ormetiké) hacia todo objeto de impulso (orektón). Y en efecto, anterior a todas estas facultades está lo 'uno

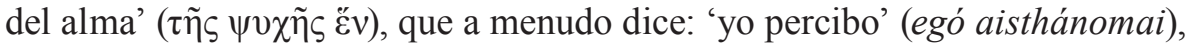
'yo calculo' (egó logídsomai), ‘yo deseo' (egó epithumó), 'yo quiero' (egó boúlomai), y que es consciente ( $\pi \alpha \rho \alpha \kappa \lambda_{0}$ ov $\theta$ ov̀v) de todas estas actividades y actúa conjuntamente con ellas. De otro modo, no tendríamos conocimiento de estas actividades, ni seríamos capaces de decir en qué difieren, si no hubiese

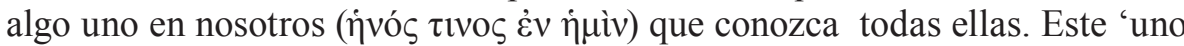
en nosotros' está por encima del sentido común, y es anterior a la opinión, al deseo y a la voluntad, y conoce todos conocimientos de estas facultades y reúne conjuntamente en sí mismo de modo indiviso todos los impulsos (oréxeis),

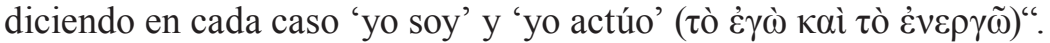

Es decir, lo "uno del alma" antecede a la unidad de cualquiera de las facultades. Y es este "uno del alma" lo que posibilita decir "yo". El "yo" por consiguiente es anterior a la autoconciencia, aunque se manifieste en ella. En resumen, en el nivel más bajo, está la conciencia por la que somos conscientes de una actividad psíquica aquí y ahora, y que parece vinculada a la atención. En un nivel superior está la autoconciencia intelectual, que define a la inteligencia. Y por último, por encima de la inteligencia, está el "uno en nosotros" o "flor del alma", que, ahora sí, constituye la unidad del ser humano, y gracias a la cual, podemos decir "yo".

En cualquier caso, la inteligencia no es sólo autoconciencia, mera presencia de la inteligencia a sí misma, según la cual decimos "yo", sino que además, como dice Aristóteles, es en cierto modo todas las cosas, o como subraya Proclo, "todo intelecto es intelectivamente todas las cosas, ya sea las antecedentes a él, ya sean las consecuentes" (Elth 173; cfr. 170). La inteligencia puede pensar todo lo real, según el modo propio de la inteligencia: desde el Uno hasta la Materia, todo es susceptible de existir en la inteligencia, no según son en sí mismos sino según el modo intelectivo. Y en tanto que cada inteligencia particular es parcial, y está ligada al tiempo y al mundo material, en esa medida la inteligencia humana entiende todas las cosas de un modo humano.

Por lo demás, la autoconciencia no es primera porque -a diferencia del "uno en nosotros"- no es productiva. El "uno en nosotros" no sólo nos permite decir "yo", sino que además no cesa nunca de producir, de crear: su constante actividad se traduce en constante productividad. Por eso, en tanto que la inteligencia depende del "uno en nosotros", en esa misma medida la inteligencia es también siempre productiva. Su actividad no es mera manifestación autoconsciente, sino también producción. Recuérdese que según el esquema neoplatónico, el universo físico deriva del alma (del Alma del Mundo en especial), y el Alma 
(todas las almas) dependen de la inteligencia y lo inteligible. Finalmente, lo inteligible procede del Uno. Por consiguiente, el alma y el universo físico dependen de la inteligencia, de un modo u otro: "Todo intelecto con el pensar hace existir a sus consecuentes; su acto creador reside en el pensar y el pensamiento en el crear" (Elth, 174). En el caso del ser humano, estas consideraciones se traducen en que el alma humana depende de su inteligencia: "toda alma deriva su existencia directamente de un intelecto" (Elth, 193).

\section{VI.2. DIETRICH DE FREIBERG ${ }^{15}$}

Como es bien sabido, son muchos y complejos los debates medievales (ss. XIII-XIV) acerca de la interpretación de De anima III, 4-5; y en general acerca de la conciencia, la autoconciencia, el intelecto agente y paciente. Estas disputas prosiguen los desarrollos de los comentadores griegos de Aristóteles (Alejandro, Temistio, Filopón, Simplicio...), así como las doctrinas de Plotino y Proclo, reformuladas en la Teología de Aristóteles y el Liber de causis y atribuidas a Aristóteles.

El interés particular de Dietrich en esta tradición filosófica reside en que es el primero que utiliza sistemáticamente los Elementos de teología de Proclo, traducidos por Moerbeke en 1268, desarrollando algunas doctrinas proclianas sobre la inteligencia y acomodándolas con las doctrinas de Aristóteles sobre la inteligencia y de Agustín sobre la mens. Por lo demás, al día de hoy, Dietrich ya no es considerado un autor menor en el panorama de la escolástica medieval, sino una figura relevante para comprender la historia de la tradición racionalista e idealista moderna, a través de la mediación del Cusano.

15 Dietrich de Freiberg (1250-1310), también llamado Dietrich de Sajonia o Theodoricus Teutonicus, realizó su actividad docente entre Alemania (donde fue Provincial superior de la O.P.) y París (Maestro de Teología en torno a 1296). Escribió sobre filosofía, teología y ciencias de la naturaleza, siguiendo el ejemplo de su maestro Alberto Magno. Sus obras están recogidas en el Corpus Philosophorum Teutonicorum Medii Aevi. Algunas de sus obras más relevantes desde un punto de vista filosófico son: De ente et essentia, De intellectu et intelligibili, De visione beatifica, De origine rerum praedicamentalium, De quiditatibus entium... Siendo discípulo de Alberto Magno, al igual que Tomás de Aquino, sin embargo criticó frontalmente diversas doctrinas tomistas, como por ejemplo la distinción real essentia-esse. Su filosofía se mueve entre el aristotelismo y el neoplatonismo (en especial, el Liber de causis y los Elementos de teología de Proclo), estableciendo una fuerte vinculación con la doctrina agustiniana de la mens. Los estudios sobre su filosofía se han multiplicado en los últimos cincuenta años, en particular a partir del escrito de K. Flasch, Kennt die mittelalterliche Philosophie die konstitutive Funktion des menschlichen Denkens? Eine Untersuchung zu Dietrich von Freiberg, Kant-Studien, 63, 182-206. Una visión general de su producción puede consultarse en: Flasch, K. (ed.), Von Meister Dietrich zu Meister Eckhart , Hamburg 1987; Kandler, K.-H., Mojsisch, B, Stammkötter, F.-B. (eds.), Dietrich von Freiberg. Neue Perspektiven seiner Philosophie, Theologie und Naturwissenschaft, Amsterdam-Philadelphia 1999. 
En la reflexión filosófica de Dietrich ocupa un lugar central la inteligencia. Interesa tener presente que en el neoplatonismo la inteligencia es la primera expresión del Uno, de un Dios primero, que en sí mismo es inexpresable e incomunicable. Por ello, es precisamente la inteligencia la que primariamente nos muestra toda la realidad, eso sí, según el modo propio de la inteligencia. Además en el neoplatonismo, a causa de esta primacía de la inteligencia, la realidad aparece ante nosotros dotada de inteligibilidad. Ciertamente caben otras lecturas del neoplatonismo, por ejemplo subrayando la inaccesibilidad del Uno y apuntando a un acceso místico a Dios, tal como ofrecen algunos textos de Eckhart. Pero lo peculiar de Dietrich es su acento -igualmente neoplatónico- en la racionalidad del ser.

No obstante, al comparar a Dietrich con Proclo, destaca ese protagonismo de la inteligencia. Pues, si en Proclo se insiste en la primacía del Uno, quedando la inteligencia por debajo incluso del Ser y de la Vida, en Dietrich hay un claro énfasis en recuperar el lugar central atribuido por Aristóteles a la inteligencia. Esta diferencia de énfasis es la que dota de originalidad a las tesis de Dietrich frente a Proclo.

En sus análisis, el intelecto agente aristotélico es presentado como el centro de la actividad intelectual, e identificado con el abditum mentis (el fondo de la mens) de Agustín. El ser humano, de este modo, se define por el intelecto agente. Éste no es una mera facultad del alma, como en Tomás de Aquino o en Alberto Magno, sino la causa esencial del alma humana, de acuerdo con lo apuntado por Proclo (cfr. Elth, 193).

Al intelecto agente se le atribuye asimismo la autoconciencia, aplicándole las afirmaciones de Proclo, recogidas en el Liber de causis: omnis intelligentia intelligit essentiam suam (XIII); omnis sciens qui scit essentiam suam est rediens ad essentiam suam reditione completa (XV). Es decir, la tradicional atribución de la autoconciencia al intelecto paciente (e incluso a la imaginación), es ahora reivindicada en primer lugar para el intelecto agente.

La actividad del intelecto agente no cesa nunca, en tanto que su esencia radica en su operación. Nunca pasa de la potencia al acto. Siempre está entendiéndose. Primum istorum, videlicet quod abditum mentis semper stat in lumine suae actualis intelligentiae, patet, quoniam, cum ipsum in sua substantia sit intellectus per essentiam, quem philosophi intellectum agentem vocant, nec alicui variationi subiciatur quantum ad exitum de potentia ad actum tam quantum ad dispositionem aliquam substantialem quam etiam accidentalem, necesse est ipsum semper fixum esse in eodem modo suae substantiae. Igitur si intelligit, semper intelligit (De visione beatifica, 1.1.2.1).

Al conocerse a sí, el intelecto agente conoce el ser: esse enim et intelligere unum ambo; et enim intellectus et ens quod in ipso idem. Ser e intelecto son lo mismo, por lo que, para el intelecto agente, conocerse es conocer el ser. Y 
al conocer el ser (ens), conoce también los otros transcendentia (unum, verum, bonum). Y conoce también su principio (en último término, Dios).

Conocer el ser implica poder conocer todas las cosas (universitas entium) dentro de sí mismo. Lo real se presenta como lo conocido en la conciencia. No conoce nada fuera de sí, y en esa medida es separado, tal como afirmaba Aristóteles. Ahora bien, todo está en el intelecto, pero lo está según el modo propio del intelecto, de acuerdo con Proclo (Elth, 103, 170). Es decir, en el intelecto agente están todas las cosas (incluido Dios), pero lo están según un modo intelectivo, y concretamente según el modo de la conciencia.

En cualquier caso, Dietrich recuerda que, tal como afirmaba Proclo (Elth 174), el intelecto es productivo: Omnis intellectus in intelligendo instituit que post ipsum, et factio in intelligere et intelligentia in facere. Esse enim et intelligere unum ambo; et enim intellectus et ens quod in ipso idem. Si igitur facit per esse, esse autem intelligere est, facit per intelligere (De intellectu et intelligibili, I.3; cfr. también III.2). Pensar es producir, y producir es pensar. La actividad intelectual no reside en la mera contemplación, sino en la producción. Ahora bien, lo que produce el intelecto humano son conceptos. Es decir, la actividad del intelecto consiste en la concepción o generación de los conceptos. Por ello, afirma Dietrich, el modo propio intelectivo como están todas las cosas en el intelecto es el ens conceptionale. En el intelecto están todas las cosas, pero no como son en sí mismas, sino según el modo concepcional propio del intelecto. En este sentido, la distinción entre el ser extramental y el ente de razón resulta secundaria, en tanto es una distinción interna al ens conceptionale. Las mismas categorías aristotélicas derivan del intelecto, tal como expone Dietrich en De origine rerum praedicamentalium. Incluso Dios aparece en el intelecto como ens conceptionale.

\section{A MODO DE CONCLUSIÓN}

Los textos de Aristóteles acerca de la conciencia en el De anima están centrados en primer lugar en III-2, en relación a la conciencia sensible. No obstante, hay algunas breves indicaciones en III- 4 y 5 que aluden a la conciencia. Además hay otras observaciones acerca de la conciencia y la autoconciencia en otros lugares, como la Metafísica, las Éticas o los Parva Naturalia.

Los comentadores griegos neoplatónicos del De anima desarrollan sus análisis precisamente en torno a III-2. Y en general subrayan que la finalidad (skopos) de Aristóteles en De anima es el estudio del alma humana, dejando por tanto fuera otras instancias superiores. De este modo, tratan de interpretar los textos sobre el intelecto "agente" o "paciente" en términos simplemente humanos, al margen por ejemplo de Metafísica XII.

Para estos comentadores, la conciencia y la autoconciencia pasan a ser cuestiones centrales, en tanto el neoplatonismo presta particular atención a la 
exigencia de conocerse a sí mismo. Por ello, someten los textos de Aristóteles a un análisis minucioso, que da lugar a diversas posturas. En líneas generales, destacan que toda forma de conciencia, incluida la conciencia sensible, depende de la actividad intelectual. Estas controversias entre los comentadores reciben un amplísimo desarrollo en las tradiciones medievales, especialmente en la filosofía árabe y latina.

Un texto especialmente relevante en la recepción de Aristóteles y sus comentadores neoplatónicos es el Liber de causis, porque integra las doctrinas aristotélicas en un contexto neoplatónico. La traducción latina de los Elementos de teología de Proclo permite comprender con mayor profundidad las tesis del De causis. Todo esto es bien conocido en Alberto Magno y sus discípulos alemanes. Dietrich de Freiberg es el primer pensador que integra sistemáticamente las doctrinas de Proclo sobre la autoconciencia con la doctrina aristotélica del intelecto, y en especial del intelecto agente. La singularidad de Dietrich estriba en subrayar en la existencia humana el papel directivo del intelecto agente, la autoconciencia y su espontaneidad creadora y autoconstituyente.

Jesús DE Garay es catedrático de filosofía en la Universidad de Sevilla.

Lineas de Investigación:

Aristóteles, aristotelismo, neoplatonismo.

Publicaciones recientes:

- DE GARAY, J., Mystery Religions and Philosophy in Proclus, en Greek Philosophy and Mystery Religions (coord. M. J. Martín Velasco), ed. Cambridge Scholars Publishing, 2016, 149-170.

- DE GARAY, J., Magia y neoplatonismo en Ficino, en: Hombre y cultura. Estudios en homenaje a Jacinto Choza (coord. F. Rdez. Valls y J. J. Padial), ed. Thémata, Sevilla, 2016, 217-232.

- DE GARAY, J., The Reception of Proclus: From Byzantium to the West. An Approach, en: Byzantine Perspectives on Neoplatonism (coord. S. Mariev), ed. W. de Gruyter, 2017, 153-174.

- DE GARAY, J., Difference and Negation. Plato's Sophist in Proclus, en: Plato's "Sophist" revisited, B. Bossi \& Th. Robinson (eds.), W. de Gruyter, Berlín, 2013, 225-245.

- DE GARAY, J., Reception of Proclus in Pletho and Ficino, en: The Theodosian Age (A.D. 379-455). Power, place, belief nd learning at the end of the Western Empire, R. García-Gasco et al. (eds.), ed. British Archaeological Reports (BAR) Series, Archeopress, 2013, 175-182.

- DE GARAY, J., Dialéctica y tiempo en Proclo, Hypnos. Revista do Centro de Estudos da Antiguidade (Saô Paulo, Brasil), 31 (2013), 172-184. 
- DE GARAY, J., The work of Proclus and its reception in Byzantium, en Cultural Transfer in Late Antiquity and Middle Ages, Ch. Burnett \& P. Mantas eds., Ed. CNERU - The Warburg Institut. Oriens Academic. Córdoba - London, 2014, 25-38.

-DE GARAY, J., La filosofía política de Proclo en ARAOS, J. y DE GARAY, J. Racionalidad política, virtudes públicas y diálogo social, Thémata Editorial, 2016, 41-60.

-DE GARAY, J., La productividad como perfección ética: Proclo y la tradición platónica, L. Flamarique (ed.), Biblioteca Nueva, 2012, 343-363.

-DE GARAY, J., Inteligencia y dialéctica en Proclo. Thémata, n.50 (2014), pp. 95-111.

Email: jgaray@us.es 